\title{
A Fully Analytical Solution of the Wellbore Stability Problem under Undrained Conditions Using a Linearised Cam-Clay Model
}

\author{
Ph. A. Charlez ${ }^{1}$ and S. Roatesi ${ }^{2}$ \\ 1 TOTAL FINA, La Défense - France \\ 2 University of Bucharest, Bucharest-Romania \\ e-mail: philippe.charlez@total.com - sroatesi@mecanica.math.unibuc.ro
}

\begin{abstract}
Résumé - Solution analytique au problème de stabilité de puits en conditions non drainées utilisant un modèle de Cam-Clay linéarisé - Cet article présente une version linéarisée du modèle de Cam-Clay intégré dans le cadre général de la théorie de la poroplasticité isotherme. La loi de comportement développée à partir du concept des contraintes effectives plastiques ne contient que deux paramètres plastiques (module d'écrouissage et pente de la droite critique). Le modèle est validé sur des chemins de contrainte homogènes (hydrostatique, triaxial drainé et triaxial non drainé) et comparé à des essais expérimentaux obtenus à partir d'essais conventionnels de laboratoire.

Ensuite, une version simplifiée du modèle est appliquée au problème du puits dans un champ de contrainte axisymétrique et en conditions non drainées. Compte tenu de la linéarité de la loi et de la connaissance a priori de la région plastique, la solution (contraintes, déformations et pression de pore) est analytique.

Les résultats montrent que pour un matériau surconsolidé (degré de consolidation inférieur à 2) la contrainte tangentielle est fortement relâchée dans la zone plastique. Plus la compressibilité du fluide est grande, plus cette relaxation est importante. En termes de stabilité, plus le fluide est compressible, plus le puits sera stable. Finalement, plus le degré de consolidation est grand, moins le puits sera stable.

Mots-clés : stabilité, puits, élasticité, non drainé.
\end{abstract}

\begin{abstract}
A Fully Analytical Solution of the Wellbore Stability Problem under Undrained Conditions Using a Linearised Cam-Clay Model - This paper presents a linearised version of the Cam-Clay model fully integrated in the scope of the general theory of poroplasticity. The constitutive law which is developed in the scope of the effective plastic stress concept, only contains two plastic parameters (hardening modulus and slope of the critical state line). To be validated, the model is integrated over homogeneous stress paths (hydrostatic, drained triaxial and undrained triaxal) then compared with experimental data issued from conventional laboratory triaxial tests.

In the second part, a simplified version of the model is applied to the wellbore boundary problem (vertical well) in an axisymmetric horizontal stress field and under undrained conditions. Given the linearity of the constitutive law and the a priori knowledge of the shape of the plastic region, the solution (stress, strain and pore pressure) is fully analytical.
\end{abstract}


The solution shows that for an overconsolidated material (overconsolidation degree less than 2) the hoop stress is strongly relaxed in the plastic zone. The higher the compressibility of the saturating fluid, the larger the relaxation of the hoop stress.

In terms of stability, the more compressible the fluid saturating the porous medium is, the more stable the well will be. Finally the larger the overconsolidation ratio is, the less stable the well will be.

Keywords: wellbore stability, undrained, plasticity.

\section{NOMENCLATURE}

$P$

$Q \quad$ stress deviator $(\mathrm{MPa})$

$p \quad$ pore pressure

$K_{B} \quad$ drained bulk modulus (MPa)

$K_{u} \quad$ undrained bulk modulus (MPa)

$E_{B} \quad$ drained Young's modulus (MPa)

$E_{u} \quad$ undrained Poisson's ratio $(\mathrm{MPa})$

$v_{B} \quad$ drained Poisson's ratio

$G \quad$ shear modulus (MPa)

$m \quad$ mass of fluid per total volume $\left(\mathrm{kg} / \mathrm{m}^{3}\right)$

$A, V$ hardening forces, hardening flux variables

$f \quad$ yield locus

$M \quad$ slope of critical state line

$H \quad$ hardening modulus (MPa)

$k \quad$ plastic bulk modulus (MPa)

$P_{\text {con }} \quad$ consolidation pressure (MPa)

$P_{c r} \quad$ critical pressure (MPa)

$p_{R} \quad$ virgin pore pressure $(\mathrm{MPa})$

$\sigma_{v} \quad$ vertical stress $(\mathrm{MPa})$

$\sigma_{h} \quad$ horizontal stress (MPa)

$B \quad$ Skempton coefficient

$\varepsilon_{k k}^{p} \quad$ mean plastic strain

$\sigma_{\rho \rho} \quad$ radial stress $(\mathrm{MPa})$

$\sigma_{\theta \theta} \quad$ hoop stress $(\mathrm{MPa})$

$R \quad$ well radius $(\mathrm{m})$

$R_{p} \quad$ plastic radius (m).

\section{INTRODUCTION}

The propping of underground cavities is one of the most basic geotechnical problem in mining engineering (stability of underground galleries) and in civil engineering (tunnelling). The objective of any stability calculation is to size a propping agent. The oil well, which is the key element in petroleum engineering, is no exception to the rule.

During the drilling phase, the well remains open hole and is propped by the drilling mud which is a complex mixture of a fluid (water or oil base) and a solid (usually bentonite or barite). Depending on the quantity of solid used, it is possible to adjust the density of the mixture over a wide range, and consequently the pressure (equal to the weight of the mud column) exerted on the borehole wall. The required density will obviously be strongly connected to the type of formation being crossed and may vary considerably during a same drilling operation.

The mud weight required to stabilise a given formation mainly depends both on the mechanical state existing before drilling (in situ stresses and virgin pore pressure) but also on the rheological behaviour of the rock concerned. From a general view point (Charlez, 1997), deep sedimentary rocks can be classified in damaging/brittle materials (cohesive sandstones and limestones) and poroplastic rocks (claystones, shales and loose sands). Due to their low shear strength, deep poroplastic materials are the most sensitive to stability problems (Veeken et al., 1989; Charlez and Heugas, 1991; Ewy, 1991; Zhou et al., 1996). A proper understanding of their rheological behaviour is consequently a key issue in view of assessing stability of deep wells.

Standards poroplastic constitutive laws are very attractive modelling tools both for the simplicity of their formulation but also for their theoretical robustness. However, does the normality hypothesis complies with experimental reality?

Poroplastic rocks are generally governed by two different elastoplastic mechanisms: a collapse mechanism purely contractant and associated with the spherical component of the stress tensor and a deviatoric mechanism governed by internal friction successively contractant, at constant plastic volume, then possibly dilatant just before macroscopic failure (Charlez and Shao, 1993). If described by a Mohr-Coulomb straight line the deviatoric mechanism (contractant or at constant plastic volume) appears incompatible with respect to the normality concept which imposes the volumetric plastic strain increment to be dilatant. The advantage of the Cambridge model resides in the successful "marriage" between these two concepts.

More widely known under the name "Cam-Clay" it was developed in the mid 1960's by Burland and Roscoe (1968). Although it was initially written for normally consolidated clay materials from surface, the very realistic physics it integrates allows adapting it to deeper materials. In the classical CamClay model, the state equations, the yield locus and the strain hardening law are non linear. We propose below a linearised version of the Cam-Clay which can be fully integrated in the case of the wellbore boundary problem. 


\section{LINEARISATION OF THE CAM-CLAY MODEL: BILINEAR MODEL}

Poroplastic rocks (Biot, 1973; Lade, 1977; Coussy,1989; Charlez, 1991; Coussy, 1995) are materials likely to undergo irreversible instantaneous deformations (no delayed effects) of both their skeleton, and their fluid mass content. Under small perturbation hypothesis, the partitioning rule of classical plastic materials (decomposition of the total strain into an elastic and a plastic part) is extended to fluid mass increments, i.e.:

$$
\mathrm{d} \varepsilon=\mathrm{d} \varepsilon^{p}+\mathrm{d} \varepsilon^{e} \quad \mathrm{~d} m=\mathrm{d} m^{p}+\mathrm{d} m^{e}
$$

$\mathrm{d} m, \mathrm{~d} m^{e}$ and $\mathrm{d} m^{p}$ being respectively total, elastic (recoverable) and plastic fluid mass increments per unit of initial volume.

\subsection{Main Hypothesis}

Two fundamental hypothesis are considered. On one hand, the plastic flow rule is considered as standard (merging of the yield locus and the plastic potential) on the other, the matrix (i.e. the grains) is assumed elastically and plastically incompressible. As shown by Coussy (1989) these hypotheses allow writing the various equations directly in terms of effective stress. Assuming a single strain hardening mecha$\operatorname{nism}(A, V)$, the plastic flow rule is written:

$$
\begin{gathered}
\mathrm{d} \varepsilon^{p}=\lambda \frac{\partial f}{\partial \sigma^{\prime}} \quad \text { and } \quad \mathrm{d} V=-\lambda \frac{\partial F}{\partial A} \\
\text { with } \sigma^{\prime}=\sigma-I p
\end{gathered}
$$

where $f$ is the yield locus and $F$ the plastic potential associated with the strain hardening mechanism (the material is not considered as standard generalised). In equations (2), $\lambda$ is the "plastic multiplier".

\subsection{Basic Equations}

In the $P, Q$ space (mean effective stress and second invariant of the stress tensor), the various equations governing the model are the following.

\subsubsection{State Law}

$$
\begin{aligned}
& P=P_{0}+K_{B}\left(\varepsilon_{k k}-\varepsilon_{k k}^{p}\right) \quad \sigma_{i j}=2 G\left(\varepsilon_{i j}-\varepsilon_{i j}^{p}\right)(i \neq j) \\
& p=p_{R}+B K_{u}\left(\varepsilon_{k k}+\frac{\mathrm{m}}{\rho_{0}}\right)
\end{aligned}
$$

where $K_{u}, K_{B}$ and $G$ are respectively the undrained bulk modulus (i.e. for $m=0$ ), the bulk drained modulus and the shear modulus (similar to that of continuous media). $B$ is the
Skempton's coefficient and $\rho_{0}$ the fluid density in the reference state.

\subsubsection{Yield Locus}

The yield locus $f$ is written as following:

$$
\begin{array}{ll}
P \leq P_{c r} & f\left(P, Q, P_{c r}\right)=Q-M P_{c r} \\
P>P_{c r} & f\left(P, Q, P_{c r}\right)=Q+M P-2 M P_{c r}
\end{array}
$$

In (4), $P_{c r}$ is the critical pressure and $M$ a material constant. Their meaning will be discussed later.

\subsubsection{Strain Hardening Law}

The strain hardening mechanism is defined by a flow variable $V$ and a strain hardening force $A$ such that:

$$
A=\frac{\partial \psi_{h a r}}{\partial V}
$$

where $\psi_{\text {har }}$ is the locked up energy per unit volume. The combination of the various plastic equations leads to the incremental law (Loret, 1987):

$$
d \sigma=\stackrel{\wedge}{e p}^{e} d \varepsilon
$$

with:

$$
{\stackrel{\ominus}{e p^{-1}}}^{=}{\stackrel{ }{B^{-1}}}^{H}\left[\frac{1}{\partial \sigma^{\prime}} \otimes \frac{\partial f}{\partial \sigma^{\prime}}\right]
$$

$\stackrel{\wedge}{=}^{e p}$ and $\stackrel{\wedge}{=}^{B}$ being respectively the elastoplastic and the drained elastic matrix. In (7), $H$ is the strain hardening modulus such that:

$$
H=\frac{\partial f}{\partial A} \frac{\partial^{2} \psi_{h a r}}{\partial V^{2}} \frac{\partial F}{\partial A}
$$

The proposed linear strain hardening law of the model is written:

$$
P_{c r}=P_{c r}^{0}+\frac{k}{2}\left(\varepsilon_{k k}^{p}-\varepsilon_{k k_{0}}^{p}\right)
$$

In (9), $k$ is a second material constants to be experimentally measured.

The yield locus of the bilinear model is studied in Figure 1. For $P>P_{c r}$, the volumetric behaviour is contractant and the yield locus moves parallel to itself such that point $A$ remains on a line of slope $M$ (called critical straight line). For $P<P_{c r}$, the yield locus is parallel to the $P$ axis and the material plastifies at constant plastic volume. We should note that the yield locus cut the $P$ axis for a value $P_{\text {con }}$ (consolidation pressure) which is equal to twice the critical pressure. The plastic formalism can be written indifferently 


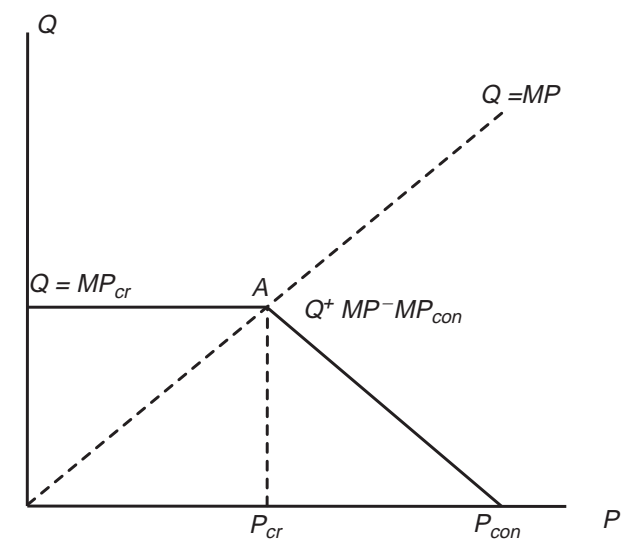

Figure 1

Bilinear model: yield locus.

using $P_{c r}$ or $P_{c o n}$. In the hardening law, $P_{c r}$ appears as a hardening force and $\varepsilon_{k k}^{p}$ as a hardening flux variable. By integrating Equation (5), we calculate the locked-up energy:

$$
\psi_{h a r}=\int P_{c r} \delta \varepsilon_{k k}^{p}=\psi_{h a r}^{0}+\frac{k}{4}\left(\varepsilon_{k k}^{p}-\varepsilon_{k k_{0}}^{p}\right)^{2}
$$

which is a quadratic function of the plastic volumetric strain. Identification of plastic flow rules (2) allows determining the plastic potential $F$ associated with the strain hardening process (the model is not standard generalised), then through Equation (8), the strain hardening modulus $H$ :

$$
\begin{array}{ll}
H=k M^{2} & \text { if } P>P_{c r} \\
H=0 & \text { if } \quad P \leq P_{c r}
\end{array}
$$

which, in both cases, is a constant. This is a direct consequence of the linear choice of the different constitutive equations. The strain hardening modulus is positive in the contractant zone (i.e., for $P>P_{c r}$ ). For $P=P_{c r}$, the strain hardening modulus is nil and the material behaves as an ideal plastic one. The locked-up energy corresponds to "grains contact energy". In the hardening phase, the grains are brought close together and the number of contacts increases, as does the locked-up energy; the material is plastically contractant and the strain hardening modulus is positive. When the representative loading point reaches the critical state line, the locked-up energy and the number of contacts remain stationary; the behaviour is then ideal plastic. For a mean stress value higher than the critical pressure, calculation of the plastic matrix is carried out by applying Equation (7) and leads to the following linear incremental law:

$$
P>P_{c r}\left\{\begin{array}{l}
\mathrm{d} \varepsilon_{k k}^{p}=\frac{M^{2}}{H} \mathrm{~d} P+\frac{M}{H} \mathrm{~d} Q \\
\mathrm{~d} \varepsilon^{p}=\frac{M}{H} \mathrm{~d} P+\frac{1}{H} \mathrm{~d} Q
\end{array}\right.
$$

$\mathrm{d} \varepsilon^{p}$ being the deviatoric strain. Unlike a purely elastic model, the deviatoric stress induces volumetric deformations during the strain hardening phase. Apart from the elastic constants $E_{B}$ and $\mathrm{v}_{B}$, the bilinear model contains 3 plastic parameters $H, M$ and $P_{c r}\left(\right.$ or $\left.P_{c o n}\right)$.

\subsection{Integration on Homogeneous Stress Paths}

For a homogeneous triaxial stress path $\left(\sigma_{1}^{\prime}, \sigma_{2}^{\prime}=\sigma_{3}^{\prime}\right)$, the incremental elastoplastic law (12) becomes:

$$
\begin{aligned}
\mathrm{d} \varepsilon_{1} & =\left[\frac{1}{E_{B}}+\frac{1}{3 H}\left(\frac{M^{2}}{3}+2 M+3\right)\right] \mathrm{d} \sigma_{1}^{\prime} \\
& +\left[\frac{-2 v_{B}}{E_{B}}+\frac{1}{3 H}\left(\frac{2 M^{2}}{3}+M-3\right)\right] \mathrm{d} \sigma_{3}^{\prime} \\
\mathrm{d} \varepsilon_{3} & =\left[-\frac{v_{B}}{E_{B}}+\frac{1}{2 H}\left(\frac{2 M^{2}}{9}+\frac{M}{3}-1\right)\right] \mathrm{d} \sigma_{1}^{\prime} \\
& +\left[\frac{1-v_{B}}{E_{B}}+\frac{1}{2 H}\left(\frac{4 M^{2}}{9}-\frac{4 M}{3}+1\right)\right] \mathrm{d} \sigma_{3}^{\prime}
\end{aligned}
$$

For a hydrostatic compression test $\left(\mathrm{d} \sigma_{1}^{\prime}=\mathrm{d} \sigma_{3}^{\prime}=\mathrm{d} P\right)$, the volumetric deformation evolves with the mean stress according to:

$$
\mathrm{d} \varepsilon_{k k}=\left[\frac{1}{K_{B}}+\frac{1}{k}\right] \mathrm{d} P
$$

The constant $k$ (introduced in the strain hardening law (9)) plays the role of a plastic bulk modulus.

The drained triaxial test, which consists of keeping the confining pressure constant $\left(\mathrm{d}_{\sigma_{3}^{\prime}}=0\right)$ under drained conditions, corresponds in the $P-Q$ diagram to a straight line of slope +3 . For such a stress path, the incremental law (13) is written:

$$
\begin{aligned}
& \mathrm{d} \varepsilon_{1}=\left[\frac{1}{E_{B}}+\frac{1}{3 H}\left(\frac{M^{2}}{3}+2 M+3\right)\right] \mathrm{d} \sigma_{1}^{\prime} \\
& \mathrm{d} \varepsilon_{3}=\left[-\frac{v_{B}}{E_{B}}+\frac{1}{2 H}\left(\frac{2 M^{2}}{9}+\frac{M}{3}-1\right)\right] \mathrm{d} \sigma_{1}^{\prime}
\end{aligned}
$$

During the strain-hardening phase, the axial stress evolves linearly versus the axial strain with a slope $\alpha$ such that:

$$
\alpha=\frac{1}{\frac{1}{E_{B}}+\frac{1}{3 H}\left(\frac{M^{2}}{3}+2 M+3\right)}
$$

As shown on Figure 2, for normal consolidation conditions, the material exhibits a strain hardening/ideal plastic behaviour whereas, for an overconsolidated material (degree of overconsolidation less than 2), an elastic/strain hardening/ideal plastic behaviour will be observed. These 


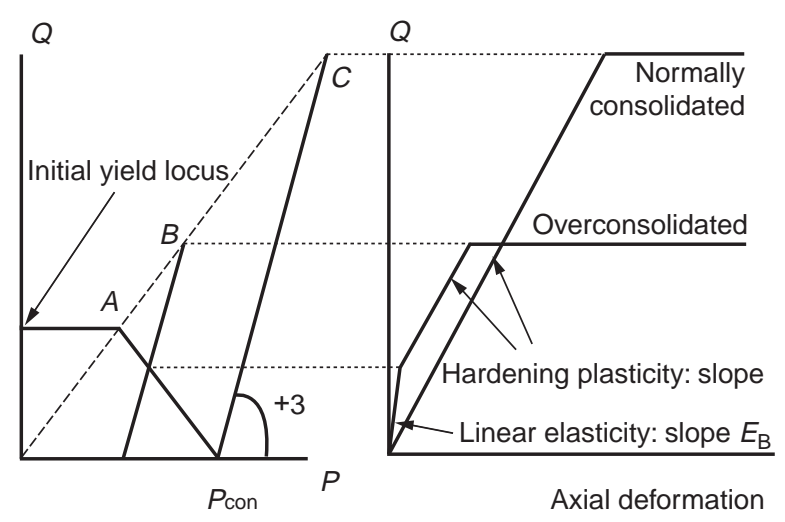

Figure 2

Behaviour of the bilinear model under drained triaxial loading.

results also demonstrate the lower rupture limit of an overconsolidated material which fails at a lower $Q$ value (compare $Q$ at $B$ and $C$ ) than a normally consolidated one.

A calibration example of the bilinear model on drained triaxial tests is presented on Figure 3 (shallow sand from Orenico Delta, Venezuela). The best fitting gives the following set of parameters: $E_{B}=125 \mathrm{MPa}, H=1000 \mathrm{MPa}$, $v_{B}=0.33$ and $M=1.16$. However, given the linearity of the constitutive equations, the model is not able to take into account the strong non linearities experienced during strain hardening.

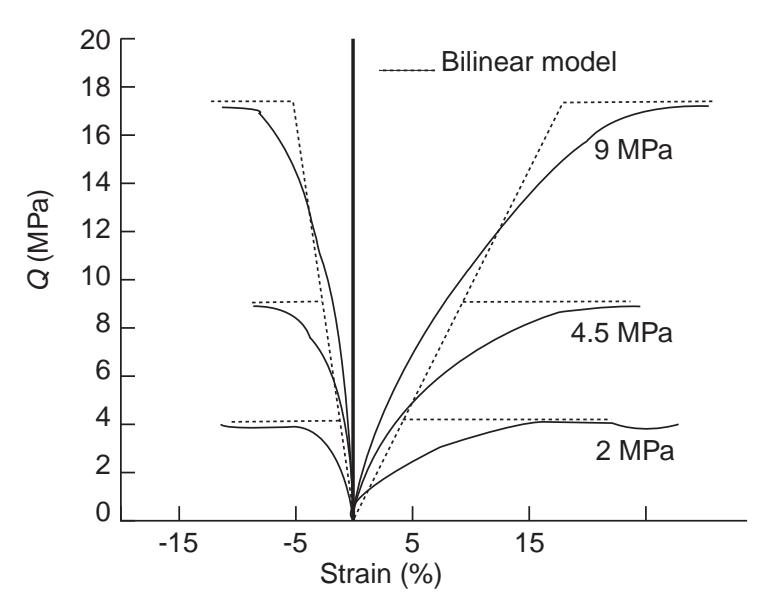

Figure 3

Validation of the bilinear model on drained tests (loose sand/Orenico, Venezuela).
In the case of an incompressible fluid, the undrained path corresponds to an isochore transformation:

$$
\mathrm{d} \varepsilon_{k k}=\left[\frac{1}{K_{u}}+\frac{M^{2}}{H}\right] \mathrm{d} P+\frac{M}{H} \mathrm{~d} Q=0
$$

In the $P-Q$ diagram, the undrained triaxial test follows a slope b such that:

$$
\beta=-\left(M+\frac{H}{M K_{u}}\right)
$$

The undrained path is therefore plastic contractant for a normally consolidated material, elastic (this time the slope is equal to the undrained Young's modulus), then contractant plastic in the case of an overconsolidated material. For materials with an overconsolidation ratio equal to 2 , the material reaches the critical state by following a purely elastic path (Fig. 4). In the $Q-\varepsilon$ diagram, the strain hardening phase follows a slope $\alpha^{\prime}$ such that:

$$
\alpha^{\prime}=\frac{1}{\frac{1}{E_{u}}+\frac{1}{3 H}\left[\frac{M^{2}}{3}+2 M+3\right]}
$$

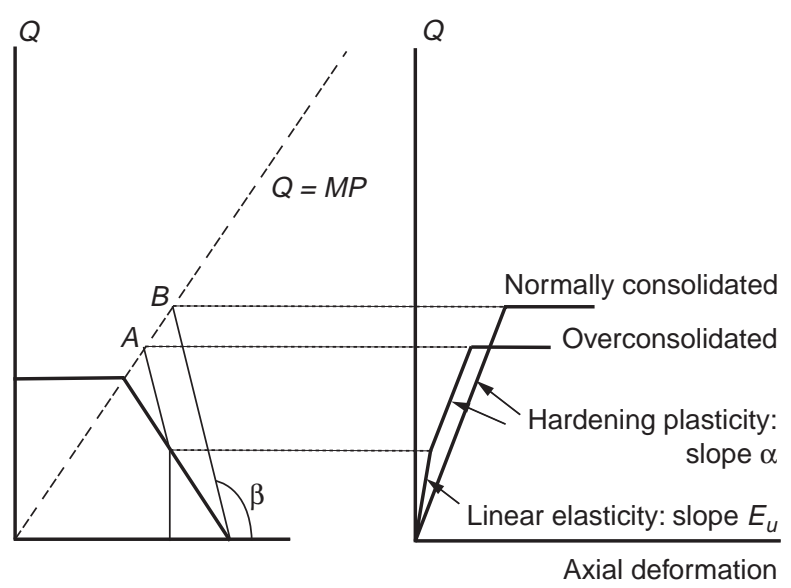

Figure 4

Behaviour of the bilinear model under undrained triaxial loading.

The fluid which contributes directly to elastic deformation under undrained conditions $\left(E_{u}\right.$ is different, depending on whether the fluid is compressible or not) plays no role in the plastic strain hardening. This is perfectly consistent with the physical aspects of strain hardening phenomena (no hardening energy is locked up in the fluid).

An example of validation (remoulded samples from Villeperdue marl) of the bilinear model on triaxial undrained tests is presented on Figure 5. The best set of parameters 


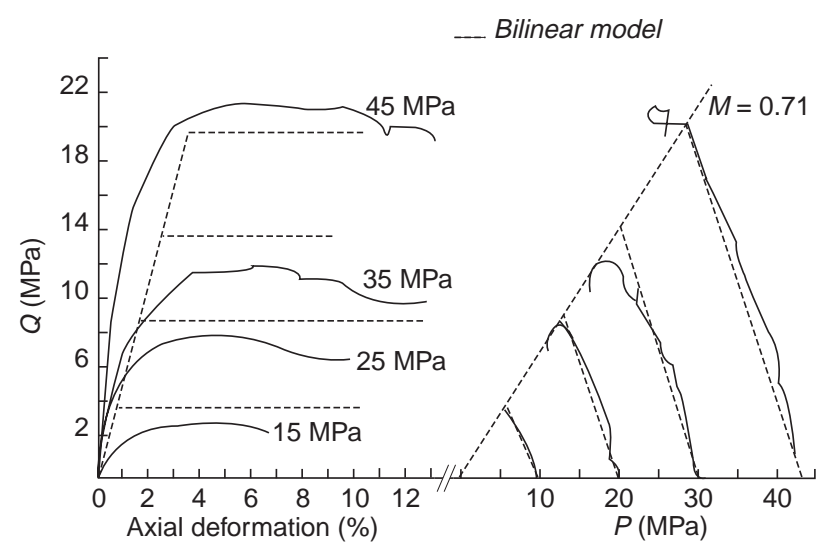

Figure 5

Validation of the bilinear model on undrained tests (remoulded specimens/Villeperdue field).

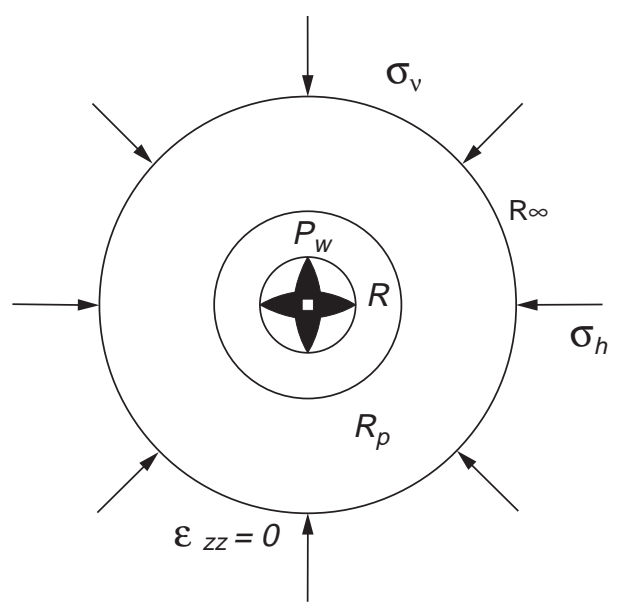

Figure 6

Well axisymmetric plane problem. obtained from these undrained tests is the following: $E_{u}=$ $2800 \mathrm{MPa}, v_{u}=0.32, H=1150 \mathrm{MPa}, M=0.71$. The elastic constants are in good agreement with the pore fluid compressibility modulus (water in this case).

In the $P-Q$ plane the model fits quite well the experimental results. However, in the stress/strain diagram due to the strong experimental nonlinearities the bilinear model does not accurately predict the experimental curves.

\section{THE PLASTIC WELLBORE PROBLEM}

Analytical solutions of plastic boundary problems are not common, as the incremental equations can only be integrated for simplified geometries. First of all the stress state being not calculated using the same equations in the plastic and the elastic zones, for an explicit solution to be found, the shape of the potential plastic zone must a priori be known. For the wellbore problem, only a circular geometry (isotropic horizontal stress field) allows obtaining analytical solutions. By contrast, if an anisotropic stress field prevails, the plastic zone being not a priori known, an implicit numerical method (finite elements) shall be used.

Although limited in their applications, these analytical solutions do have the advantage of revealing how elastoplastic parameters act on stability.

\subsection{Definition of the Problem}

Let us consider an infinite circular domain initially loaded (Fig. 6) at infinity by an isotropic horizontal geostatic stress $\sigma_{h}$ and a vertical geostatic stress $\sigma_{v}$. Before drilling, the pore pressure in the whole domain is uniform and equal to $p_{R}$.

At time $t=0$, an infinite cylinder of radius $R$ is instantaneously drilled parallel to $z$ axis. It is filled with a fluid of the same nature as that saturating the porous medium but at a different pressure $p_{w}$. Since the borehole is assumed to be infinitely long parallel to its axis, calculation of the perturbation can be performed under plane strain hypothesis $\left(\varepsilon_{z z}=0\right)$.

The boundary conditions can be summarised as follows:

$$
\begin{aligned}
t \leq 0 \rightarrow & p(\rho)=p_{R} \forall \rho & \\
t>0 \rightarrow & \sigma_{\rho \rho}(\rho=R, t)=p_{w} & \sigma_{\rho \rho}(\rho=\infty, t)=\sigma_{h} \\
& p(\rho=R, t)=p_{w} \quad & p(\rho=\infty, t)=p_{R} \\
& \varepsilon_{z z}=0 &
\end{aligned}
$$

The resolution of the undrained problem is presented in Appendix. We discuss below the main results.

\subsection{Discussion of the Results}

The analytical solution is studied below using the following set of parameters. For simplicity, calculations are performed considering that the initial pore pressure $p_{R}$ is equal to 0 .

TABLE 1

Set of parameters used to study the wellbore boundary problem

\begin{tabular}{c|c|c}
\hline$E_{B}$ & $\mathrm{MPa}$ & 10000 \\
\hline$v_{B}$ & - & 0.3 \\
\hline$\phi$ & - & 0.1 \\
\hline$R_{p}$ & - & 5 \\
\hline$\sigma_{h}$ & $\mathrm{MPa}$ & 30 \\
\hline$\sigma_{v}$ & $\mathrm{MPa}$ & 40 \\
\hline$p_{R}$ & $\mathrm{MPa}$ & 0 \\
\hline$P_{c}^{0}$ & $\mathrm{MPa}$ & 40 \\
\hline
\end{tabular}




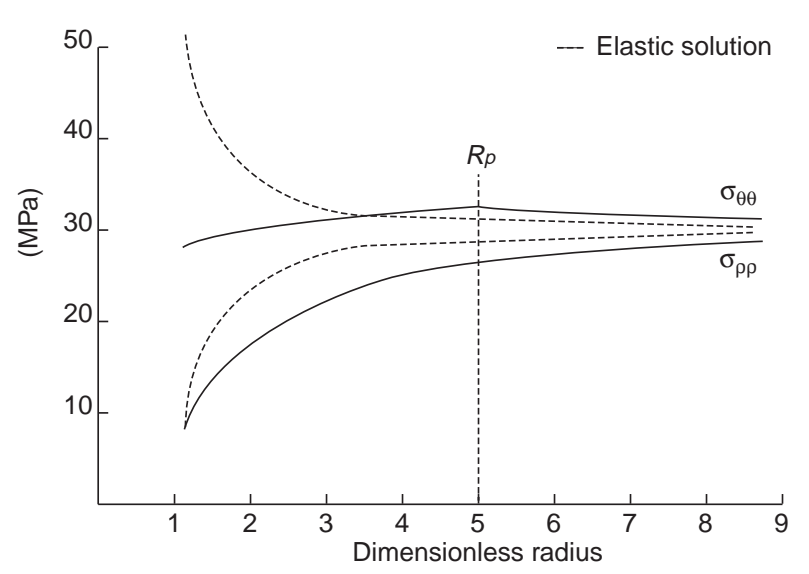

Figure 7

Stress state around the borehole. Comparison with the elastic solution $(M=0.7, H=100 \mathrm{MPa}, B=0)$.

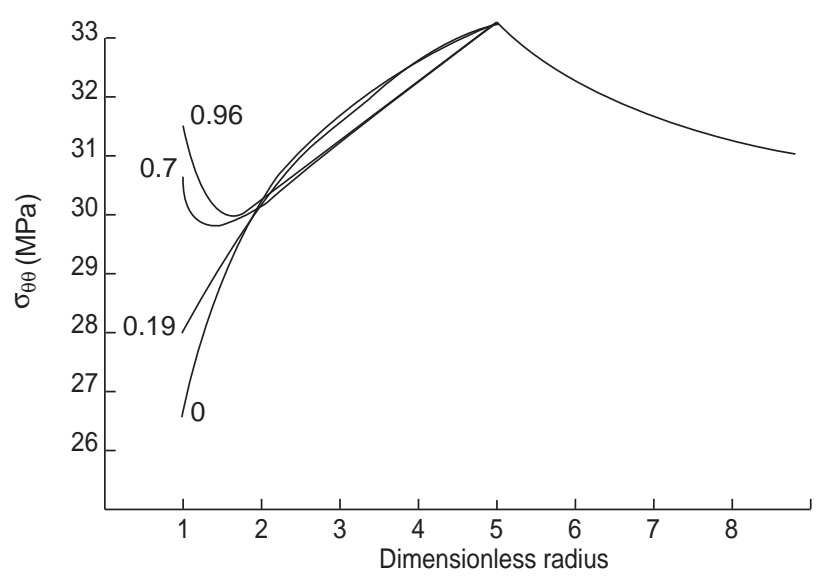

Figure 8

Effect of Skempton's coefficient on hoop stress relaxation $(M=0.7, H=100 \mathrm{MPa})$.
As pointed out on Figure 7 a substantial relaxation of the hoop stress occurs in the strain hardened zone with respect to the elastic solution (dashed lines). However, the radial component is only slightly affected by plasticity. That result is caused by the fact that the radial stress is imposed on both boundaries (well pressure for the internal boundary, geostatic stress at infinity).

The effect of the Skempton's coefficient on hoop stress relaxation is discussed on Figure 8. The lower the Skempton's coefficient, the more the hoop stress is relaxed in the well vicinity. In other words, the higher the fluid compressibility ( $B$ close to 0$)$, the higher the hoop stress relaxation.

For the same reasons as previously, Skempton's coefficient has a marginal effect on the radial component. In fact, for infinitely compressible fluids, the undrained solution converges towards the drained solution. Drained and undrained solutions characterises respectively instantaneous and very long-term behaviours of the structure.

As pointed on Figure 9a, for similar boundary conditions (same well pressure), a low $H$ value tends on one hand to increase the extension of the plastic zone (larger plastic radius), but also to further relax the hoop stress. It is thus possible, depending on the value of the strain hardening modulus, to obtain either a decreasing hoop stress (high values of $H$ ) or a peak opposite the plastic radius (low $H$ values). Finally, $M$ (slope of critical straight line) has a similar effect on the hoop stress: a low value of $M$ tends to increase the extension of the plastic zone and further relaxes the hoop stress (Fig. 9 b).

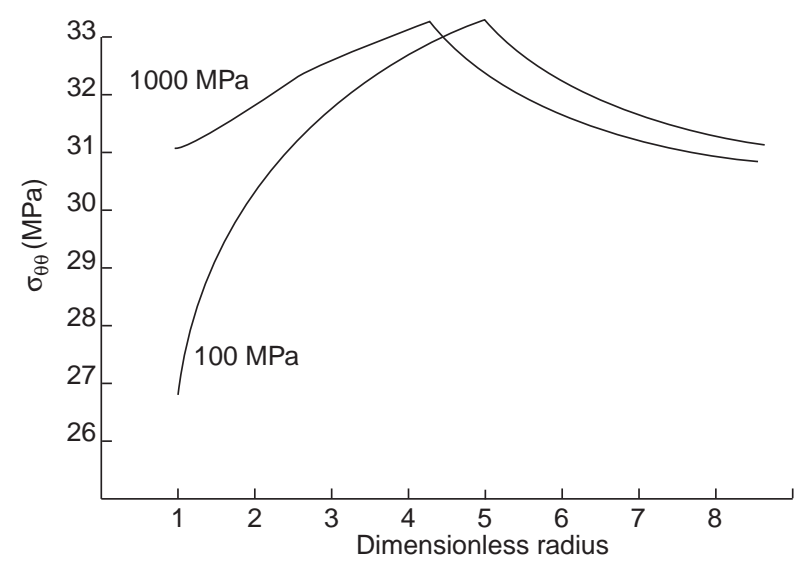

Figure 9a

Influence of $H(M=0.7)$ on hoop stress relaxation $(B=0)$.

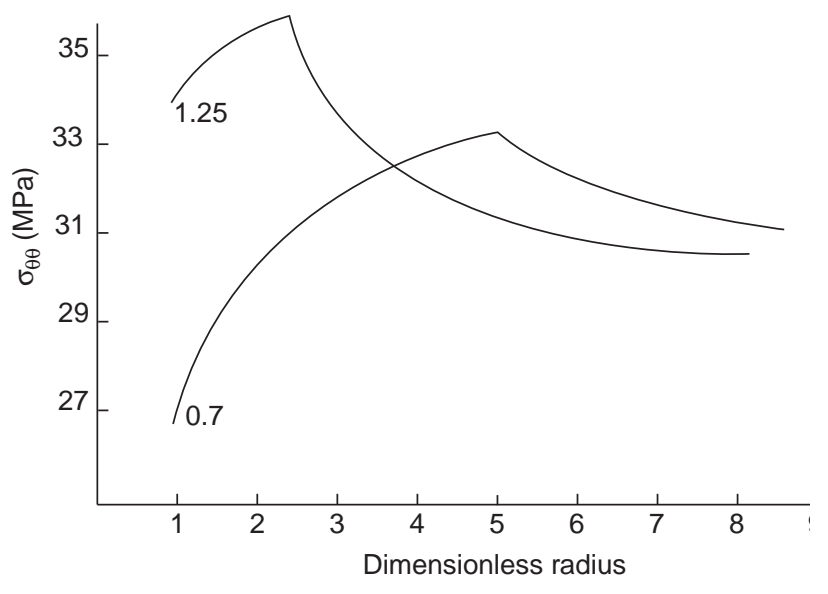

Figure $9 b$

Influence of $M(H=100 \mathrm{MPa})$ on hoop stress relaxation $(B=0)$. 
With respect to Equation (A9), the pressure response is the sum of two terms respectively connected to the mean total stress variation (Skempton's effect) and the plastic volumetric strain.

In fact, these two effects play in opposite sense (Fig. 10), as the mean stress and the plastic volumetric strain are respectively minimum and maximum at the borehole wall. In most cases, the term connected to the plastic volumetric strain is the largest. Consequently, a maximum pore pressure at the borehole wall will prevail followed by a

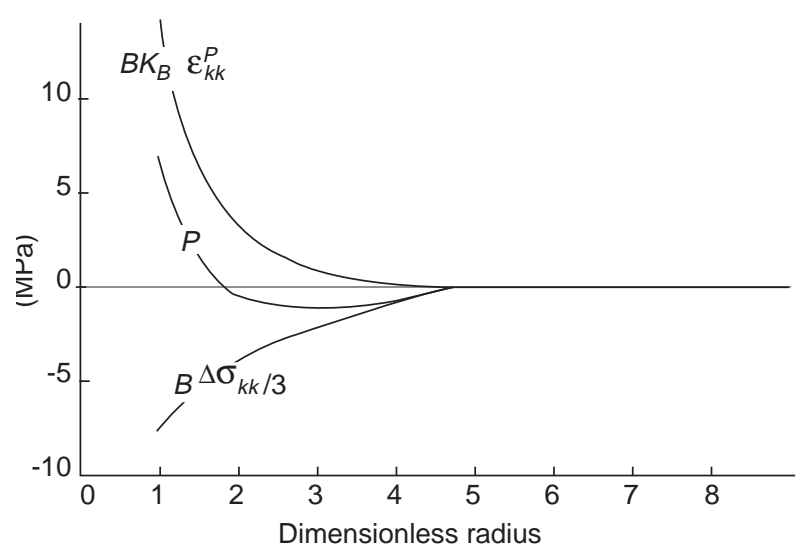

Figure 10

Decomposition of the undrained pressure response.

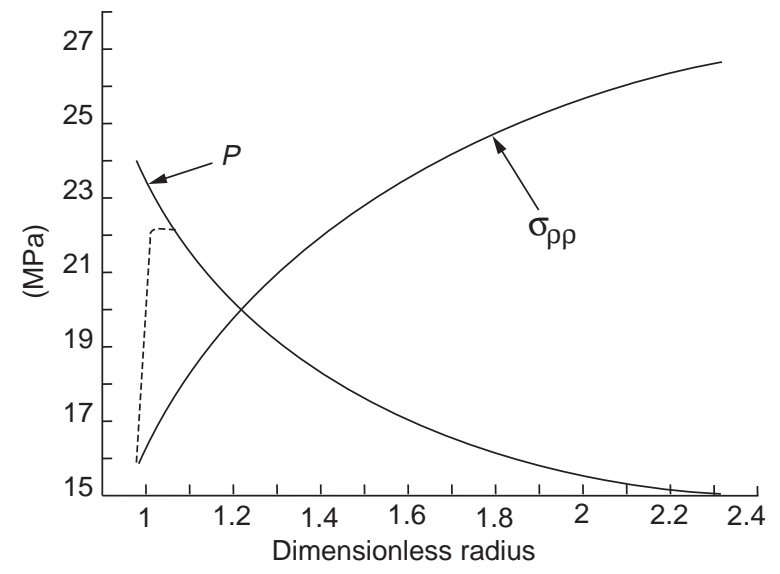

Figure 11

Incompatibility between boundary conditions at the borehole wall and the undrained response. decrease which is nullified opposite the plastic radius. In the case of low compressible fluids, however, the term related to the mean stress can, at a certain distance away from the borehole, become predominant. The pressure curve then passes through a slightly marked minimum.

Nevertheless (Detournay and Cheng, 1988), the undrained pressure response does not properly respect the boundary condition imposed at the borehole wall. Physically two situations are possible. If the borehole wall is impermeable (oil-base mud, perfect cake) or the pressure in the borehole is not applied directly by the fluid (but through a rubber membrane such as a packer for instance), the downhole pressure will not necessarily be equal to the pore pressure at the wall. On the contrary, in the case of a permeable borehole, the undrained solution at the borehole wall is no longer valid. Instantaneously, the pore pressure achieves equilibrium with the condition imposed at the boundary and the pressure exhibits a peak lying inside the block (Fig. 11).

\subsection{Critical Instantaneous Pressure}

The critical pressure corresponds to the well pressure for which the critical state is reached that is:

$$
\begin{aligned}
& \frac{1}{\sqrt{2}}\left(\sigma_{\theta \theta}-\sigma_{\rho \rho}\right) \\
& =\mathrm{M}\left[\frac{\sigma_{v}-2 v_{B} \sigma_{h}+\left(1+v_{B}\right)\left(\sigma_{\theta \theta}+\sigma_{\rho \rho}\right)}{3}-p\right]
\end{aligned}
$$

For a given $M$ value, the lower the Skempton coefficient, the lower the critical instantaneous pressure (Fig. 12a). In other words, the more compressible the fluid saturating the porous medium is, the more stable the well will be. A zero Skempton coefficient corresponding to the perfectly drained case the undrained solution is less stable than the drained solution. For more complex constitutive laws (nonlinear Cam-Clay, Laderock) and depending on the rock permeability, drainage (that is time) have sometimes a negative effect on stability.

The critical pressure decreases when $M$ increases (more stable well) but, for a same $M$ value, the critical pressure increases with the hardening modulus $H$ (Fig. 12b). If critical pressures are compared with those issued from a purely elastic solution, the latter is more pessimistic. With respect to the hardening modulus $H$, the elastic solution appears as a limit of the elastoplastic solution for which the strain hardening modulus is infinite. Generally speaking, plastic hardening plays a favourable role on stability.

\subsection{Impact of the Consolidation Degree}

For a normally consolidated material the initial consolidation pressure is equal to the mean effective stress, i.e.:

$$
P_{c o n}^{0}=\frac{\sigma_{v}+2 \sigma_{h}}{3}
$$




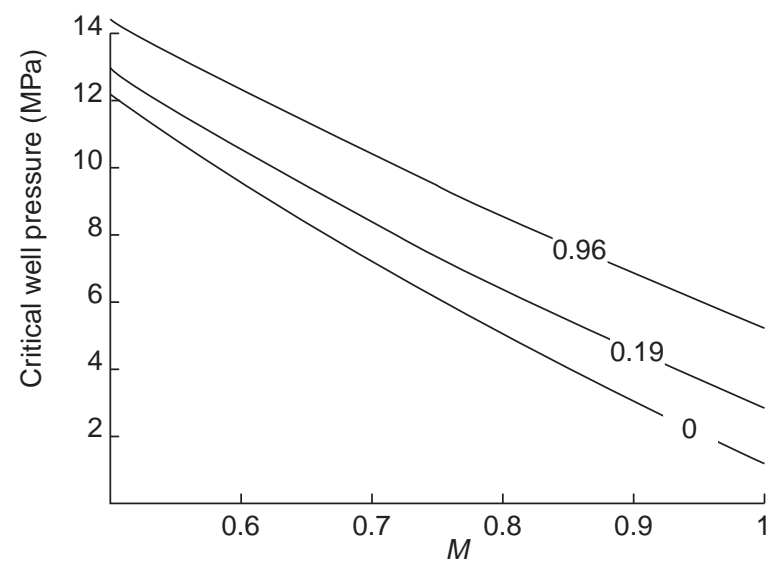

Figure 12a

Critical instantaneous pressure versus M; effect of Skempton coefficient $(H=1000)$.

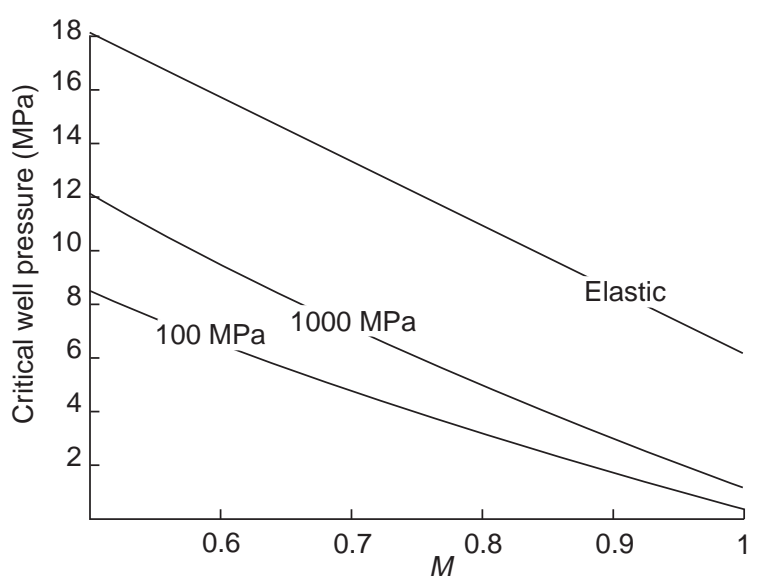

Figure $12 b$

Critical instantaneous pressure versus M; effect of Skempton coefficient $H(B=1000)$.
In such a material, the plastic radius moves out to infinity. In other words, all the points are initially plastically admissible and the material hardens as soon as the well pressure drops below the horizontal stress $\sigma_{h}$.

As pointed out on Figure 13 (the vertical stress is used as a single parameter), the critical pressure is an increasing function of the over consolidation ratio. In other words, the larger the over consolidation ratio is the less stable the well will be.

Finally, the plastic radius (corresponding to the critical pressure) strongly decreases versus the consolidation ratio, the normally consolidated material being the case for which the plastic radius is infinite.

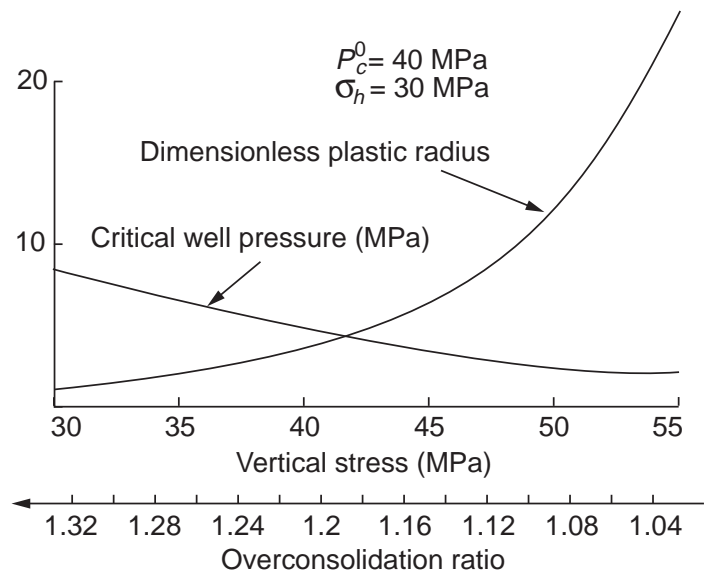

Figure 13

Influence of over consolidation ratio on critical pressure and plastic radius $(B=0)$.

\section{CONCLUSION}

In a range of confining pressures of several tens of MPa, soft deep materials (particularly shales and unconsolidated sands) clearly exhibit a ductile/plastic behaviour. Modelisation of such materials is therefore of a strategic importance to predict stability of oil wells. Complex elastoplastic models including several hardening mechanisms generally require heavy numerical tools (finite elements). Consequently, simplified constitutive laws can be very usefull in day to day engineering.

Such simplified laws have to be developped in the scope of the Terzaghi's effective stress concept which allows solving the poroplastic problem as an equivalent dry problem. This concept is only valid for an incompressible rock matrix (both elastically and plastically) and an associated plastic flow rule.

For any boundary problem to be solved analytically, the shape of the plastic zone has first of all to be a priori known. In the case of the wellbore boundary problem, it will only be possible for plane strain axisymmetric cases (vertical well and horizontal isotropic stress) for which the plastic zone is a circular ring. Secondly, to be analytically integrated, the constitutive relations will be linear (linear relationship between stress and strain, linear yield locus, linear hardening law).

A linearised Cam-Clay has been proposed in the scope of this paper. It has been validated on homogeneous drained and undrained triaxial tests then a simplified version has been integrated in the case of the undrained boundary well problem. The main results can be summarised as follows:

- compared to a purely elastic solution, a strong relaxation of the hoop stress is observed in the plastic zone;

- by contrast, the radial stress (for which the value is imposed on the two boundaries) is only slightly affected by plasticity; 
- plastic hardening plays in favour of stability: the higher the hardening modulus, the less the hoop stress relaxation is and the less the well will be stable;

- the elastic solution can be considered as the limiting case of an elastoplastic model for which the hardening modulus is infinite. For this reason, the elastic solution will always be the more pessimistic and will always overestimate the critical well pressure;

- a low fluid compressibility decreases the relaxation of the hoop stress in the well vicinity and plays a negative role on stability;

- the critical pressure is an increasing function of the over consolidation ratio. In other words, the larger the over consolidation ratio is, the less stable the well will be

\section{ACKNOWLEDGMENTS}

This work has been performed in the scope of the Star Artep project TOTAL FINA. The authors thank TOTAL for allowing to publish this paper.

\section{REFERENCES}

1 Charlez, P.A. (1997) Impact of Constitutive Laws on Wellbore Stability. SPE Drill. and Compl .Jour.

2 Veeken, C., Walters, J.V, Enter, C.J. and Davies, D. (1989) Use of Plasticity Models for Predicting Borehole Stability. Symposium Rock at Great Depth, Pau 1989, A.A. Balkema.

3 Charlez, P.A. and Heugas, O. (1991) Evaluation of Optimal Mud Weight in Soft Shale Levels. Proceedings of the 32nd US Symposium Rock Mechanics as a Multidisciplinary Science, Norman, A.A. Balkema.

4 Ewy, R.T. (1991) 3D Stress Effects in Elastoplastic Wellbore Failure Models. Proceedings of the 32nd US Symposium Rock Mechanics as a Multidisciplinary Science, Norman, A.A. Balkema.
5 Zhou, S., Hillis, R. and Sandiford, M. (1996) A Supplement to a Study of the Design of Inclined Wellbores with Regard to Both Mechanical Stability and Fracture Insertion. Jour. Appl. Geophy., 36, 145-147.

6 Biot, M. (1973) Nonlinear and Semilinear Rheology of Porous Solids. Jour. Geophy. Res., 78, 23.

7 Lade, P.V. (1977) Prediction of Undrained Behaviour of Sand. Jour. of the Geot. Eng. Div.

8 Coussy, O. (1989) General Theory of Thermoporoelastoplasticity. Transport in Porous Media.

9 Coussy, O. (1995) Mechanics of Porous Media, John Wiley \& Sons.

10 Charlez, P.A. (1991) Rock Mechanics Vol. I Theoretical Fundamentals, Éditions Technip, Paris.

11 Loret, B. (1987) Élastoplasticité à simple potentiel. Manuel de rhéologie des géomatériaux, ENPC Press.

12 Charlez, P.A. and Shao, J.F. (1993) Mechanical Behaviour of Soft Deep Rocks. Geotechnical Engineering of Hard Soils Soft Rocks, Athens 20-23 Sept. 1993, A.A. Balkema.

13 Burland, I.B. and Roscoe, K.H. (1968) On the Generalised Stress Strain Behaviour of Wet Clay. Engineering Plasticity, Cambridge Heyman-Leckie.

14 Addis, M.A. and Wu, B. (1993) The Role of Intermediate Principal Stress in Wellbore Stability Studies: Evidence from Hollow Cylinder Tests. Int. Jour. Rock Mech. Min. Sc. \& Geomech. Abstract, 30.

15 Guenot, A. (1987) Contraintes et ruptures autour des forages pétroliers. Proc. of the 6th ISRM Congr., Montreal, A.A. Balkema.

16 Jaeger, J.C. and Cook, N.G.W. (1979) Fundamentals of Rock Mechanics. McGraw Hill.

17 Graziani, A. and Ribacchi, R. (1993) Critical Conditions for a Tunnel in a Strain Softening Rock. Assessment and Prevention of Failure Phenomena in Rock Engineering, Istanbul. A.A. Balkema.

18 Detournay, E. and Cheng, A.H-D (1988) Poroelastic Response of a Borehole in a Nonhydrostatic Stress Field. Int. Jour. Rock Mech. Min. Sc.\& Geomech. Abstract, 25, 3, 171-182.

Final manuscript received in July 1999 


\section{APPENDIX}

\section{Resolution of the Wellbore Boundary Problem}

Before drilling the whole domain is supposed to be in an elastic state (over consolidated material). Depending on the values of the well pressure, the points located around the hole become plastically admissible. Given the axisymmetrical character of the problem, the plastic zone is a circular ring of radius $R_{p}$. Considering the partitioning rule and the plane strain hypothesis, it can be assumed that both axial elastic and plastic deformations are null i.e.:

$$
\varepsilon_{z z}=0=\varepsilon_{z z}^{e}+\varepsilon_{z z}^{p} \Rightarrow \varepsilon_{z z}^{e}=\varepsilon_{z z}^{p}=0
$$

On one hand, the condition (A1) on the elastic deformation allows calculating the axial stress required to ensure plane strain condition i.e.:

$$
\begin{aligned}
\varepsilon_{z z} & =0 \Rightarrow \sigma_{z z}^{\prime}=\sigma_{v}^{\prime}+\Delta \sigma_{z z}^{\prime} \\
& =\sigma_{v}^{\prime}-2 v_{B} \sigma_{h}^{\prime}+v_{B}\left(\sigma_{\rho \rho}^{\prime}+\sigma_{\theta \theta}^{\prime}\right)
\end{aligned}
$$

Consequently, the mean effective stress is written:

$$
P=\frac{1}{3}\left[\sigma_{v}^{\prime}-2 v_{B} \sigma_{h}^{\prime}+\left(1+v_{B}\right)\left(\sigma_{\theta \theta}^{\prime}+\sigma_{\rho \rho}^{\prime}\right)\right]
$$

On the other hand, condition (A1) on the plastic axial deformation implies that the yield locus is independent of $\sigma_{z z}^{\prime}$. In the general case, $Q$ is written:

$$
Q=\frac{1}{\sqrt{2}} \sqrt{\left(\sigma_{\theta \theta}^{\prime}-\sigma_{\rho \rho}^{\prime}\right)^{2}+\left(\sigma_{\rho \rho}^{\prime}-\sigma_{z z}^{\prime}\right)^{2}+\left(\sigma_{z z}^{\prime}-\sigma_{\theta \theta}^{\prime}\right)^{2}}
$$

However, borehole stability only slightly depends on the intermediate stress (Addis and Wu, 1993). As in many cases this is confirmed by the shape of the cuttings (Guenot, 1987) $\sigma_{z z}^{\prime}$ is the intermediate stress, (A4) can be rewritten:

$$
Q=\frac{1}{\sqrt{2}}\left(\sigma_{\theta \theta}^{\prime}-\sigma_{\rho \rho}^{\prime}\right)
$$

Considering (A3) and (A5), the yield locus (4) can be simplified (assuming a degree of consolidation less than 2):

$$
\begin{aligned}
f\left(\sigma_{\rho \rho}^{\prime}, \sigma_{\theta \theta}^{\prime}, P_{c o n}\right)= & \frac{1}{\sqrt{2}}\left(\sigma_{\theta \theta}^{\prime}-\sigma_{\rho \rho}^{\prime}\right) \\
& +\frac{M}{3}\left[\sigma_{v}^{\prime}-2 v_{B} \sigma_{h}^{\prime}+\left(1+v_{B}\right)\left(\sigma_{\theta \theta}^{\prime}+\sigma_{\rho \rho}^{\prime}\right)\right](\text { A6 }) \\
& -M P_{c o n}
\end{aligned}
$$

The strain hardening law (9) which depends only on the volumetric strain remains unchanged. Expression (A6) allows recalculating the incremental law (7) (in its inverse form) which according to the incompressibility of the matrix can be directly expressed in terms of effective plastic stresses, i.e.:

$$
\begin{aligned}
& \mathrm{d} \sigma_{\rho \rho}-\mathrm{d} p=\frac{1}{\operatorname{det}|\boldsymbol{A}|}\left[A_{11} \mathrm{~d} \varepsilon_{\rho \rho}+A_{12} \mathrm{~d} \varepsilon_{\theta \theta}\right] \\
& \mathrm{d} \sigma_{\theta \theta}-\mathrm{d} p=\frac{1}{\operatorname{det}|\boldsymbol{A}|}\left[A_{21} \mathrm{~d} \varepsilon_{\rho \rho}+A_{22} \mathrm{~d} \varepsilon_{\theta \theta}\right]
\end{aligned}
$$

with:

$$
\begin{aligned}
& A_{11}=\frac{1}{E^{\prime}}+\frac{1}{H}\left[\frac{M\left(1+v_{B}\right)}{3}+\frac{1}{\sqrt{2}}\right]^{2} \\
& A_{12}=A_{21}=\frac{v^{\prime}}{E^{\prime}}-\frac{1}{H}\left[\frac{M^{2}\left(1+v_{B}\right)^{2}}{9}-\frac{1}{2}\right] \\
& A_{22}=\frac{1}{E^{\prime}}+\frac{1}{H}\left[\frac{M\left(1+v_{B}\right)}{3}-\frac{1}{\sqrt{2}}\right]^{2} \\
& \text { with } E^{\prime}=\frac{E_{B}}{1-v_{B}^{2}} \text { and } v^{\prime}=\frac{v_{B}}{1-v_{B}}
\end{aligned}
$$

\section{Undrained Response}

The undrained poroplastic response is the sum of the Skempton's effect and a contribution related to the plastic volumetric strain. In the case of an elastically and plastically incompressible matrix the variation in pore pressure is written (Charlez, 1997):

$$
\mathrm{d} p=\frac{B\left(1+\mathrm{v}_{u}\right)\left(\mathrm{d} \sigma_{\rho \rho}+\mathrm{d} \sigma_{\theta \theta}\right)}{3}+B K_{B}\left(\mathrm{~d} \varepsilon_{\rho \rho}+\mathrm{d} \varepsilon_{\theta \theta}\right)
$$

After elimination of $\mathrm{d} p$ between (A7) and (A9), one obtains:

$$
\begin{aligned}
& \mathrm{d} \sigma_{\rho \rho}=\frac{1}{\operatorname{det}|\boldsymbol{A}|}\left[C_{11} \mathrm{~d} \varepsilon_{\rho \rho}+C_{12} \mathrm{~d} \varepsilon_{\theta \theta}\right] \\
& \mathrm{d} \sigma_{\theta \theta}=\frac{1}{\operatorname{det}|\boldsymbol{A}|}\left[C_{21} \mathrm{~d} \varepsilon_{\rho \rho}+C_{22} \mathrm{~d} \varepsilon_{\theta \theta}\right]
\end{aligned}
$$

where the new coefficients are such that:

$$
\begin{aligned}
& C_{11}=\frac{\left(k_{1} B_{11}+k_{2} B_{12}\right) \operatorname{det}|\boldsymbol{A}|}{\left(k_{1}^{2}-k_{2}^{2}\right)} \\
& C_{12}=\frac{\left(k_{1} B_{12}+k_{2} B_{22}\right) \operatorname{det}|\boldsymbol{A}|}{\left(k_{1}^{2}-k_{2}^{2}\right)} \\
& C_{21}=\frac{\left(k_{2} B_{11}+k_{1} B_{21}\right) \operatorname{det}|\boldsymbol{A}|}{\left(k_{1}^{2}-k_{2}^{2}\right)} \\
& C_{22}=\frac{\left(k_{2} B_{12}+k_{1} B_{22}\right) \operatorname{det}|\boldsymbol{A}|}{\left(k_{1}^{2}-k_{2}^{2}\right)}
\end{aligned}
$$


with:

$$
\begin{array}{ll}
k_{1}=\frac{3-B\left(1+v_{u}\right)}{3} & k_{2}=\frac{B\left(1+v_{u}\right)}{3} \\
B_{i j}=\frac{A_{i j}}{\operatorname{det}|\boldsymbol{A}|}+B K_{B} & B_{i j}=B_{j i}
\end{array}
$$

In the $B_{i j}$ coefficients, the first term characterises the incremental skeleton stiffness (elastic and plastic), whereas the second only depends on the fluid (via the Skempton's coefficient $B$ ). Note that unlike matrix $\mathbf{A}$, matrix $\mathbf{C}$ is not symmetrical.

If the material is assumed over consolidated, the whole domain is initially in elastic state. In that case, the initial consolidation pressure $P_{c o n}^{0}$ is greater than the initial mean stress, i.e.:

$$
P_{c o n}^{0}>\frac{\sigma_{v}^{\prime}+2 \sigma_{h}^{\prime}}{3}
$$

The analysis will be restricted to over consolidation ratio of less than 2. Prior to the appearance of the plastic zone, the stress state is purely elastic that is (Jaeger and Cook, 1979):

$$
\begin{aligned}
& \sigma_{\rho \rho}^{\prime}=\sigma_{h}^{\prime}+K_{2} \frac{R^{2}}{\rho^{2}} \\
& \sigma_{\theta \theta}^{\prime}=\sigma_{h}^{\prime}-K_{2} \frac{R^{2}}{\rho^{2}}
\end{aligned}
$$

$K_{2}$ being a constant to be determined from boundary conditions. It is interesting to note that the elastic stresses do not induce any variation in mean stress and consequently no undrained response (for a purely elastic path, the pore pressure remains equal to the virgin value $p_{R}$ ). The structure is loaded by decreasing gradually the well pressure (while keeping the geostatic stress $\sigma_{h}$ constant) until a plastic zone of radius $R_{p}$ appears and extends. For $\rho=R_{p}$, the stress field is both elastically - the stresses are compatible with Equation (A14) - and plastically admissible (these same stresses verify the yield locus (A6)) i.e.:

$$
\begin{aligned}
& {\overline{\sigma^{\prime}}}_{\theta \theta}=2 \sigma_{h}^{\prime}-{\overline{\sigma^{\prime}}}_{\rho \rho} \\
& {\overline{\sigma^{\prime} \rho}}_{\rho \rho}^{\prime}=\sigma_{h}^{\prime}+\frac{M}{\sqrt{2}}\left[\frac{\sigma_{v}^{\prime}+2 \sigma_{h}^{\prime}}{3}-P_{c o n}^{0}\right]
\end{aligned}
$$

The overlying stresses are called "initial effective plastic stresses", as they are associated with the current plastic extension. They are independent of the plastic radius. Let us note that the radial stress increasing versus radial distance, the appearance of a plastic ring is subjected to the condition:

$$
p_{w}<\bar{\sigma}_{\rho \rho} \rightarrow p_{w}<\sigma_{h}+\frac{M}{\sqrt{2}}\left[\frac{\sigma_{v}^{\prime}+2 \sigma_{h}^{\prime}}{3}-P_{c o n}^{0}\right]
$$

otherwise the whole domain remains in a purely elastic state. In the plastic ring, the total stresses will be such that:

$$
\begin{aligned}
& \sigma_{\rho \rho}=\bar{\sigma}_{\rho \rho}+d \sigma_{\rho \rho} \\
& \sigma_{\theta \theta}=\bar{\sigma}_{\theta \theta}+d \sigma_{\theta \theta}
\end{aligned}
$$

where the increments are consistent with (A10). As for stresses, the initial plastic strains can be calculated from Hooke's law, i.e. ${ }^{1}$ :

$$
\begin{aligned}
& \bar{\varepsilon}_{\rho \rho}=-\frac{1+v^{\prime}}{E^{\prime}}\left(\sigma_{h}-\bar{\sigma}_{\rho \rho}\right) \\
& \bar{\varepsilon}_{\theta \theta}=\frac{1+v^{\prime}}{E^{\prime}}\left(\sigma_{h}-\bar{\sigma}_{\rho \rho}\right)
\end{aligned}
$$

whereas in the plastic ring they are expressed as follows:

$$
\begin{aligned}
\varepsilon_{\rho \rho} & =\bar{\varepsilon}_{\rho \rho}+\mathrm{d} \varepsilon_{\rho \rho} \\
\varepsilon_{\theta \theta} & =\bar{\varepsilon}_{\theta \theta}+\mathrm{d} \varepsilon_{\theta \theta}
\end{aligned}
$$

\section{Calculation of Stresses and Strains}

Replacing (A10), (A17), (A18) and (A19) in the equilibrium equation:

$$
\frac{\partial \sigma_{\rho \rho}}{\partial \rho}+\frac{\sigma_{\rho \rho}-\sigma_{\theta \theta}}{\rho}=0
$$

and taking into account the compatibility equations:

$$
\varepsilon_{\rho \rho}=\frac{\partial u}{\partial \rho} \quad \varepsilon_{\theta \theta}=\frac{u}{\rho}
$$

we get the following differential equation:

$$
C_{11}\left[\frac{\partial^{2} u}{\partial \rho^{2}}+\frac{1}{\rho} \frac{\partial u}{\partial \rho}\right]-C_{22} \frac{u}{\rho^{2}}=-\frac{m\left(\sigma_{h}-\bar{\sigma}_{\rho \rho}\right)}{E^{\prime}} \frac{1}{\rho}
$$

with $m=-2 E^{\prime}\left[\operatorname{det}|A|+\frac{1+v^{\prime}}{2 E^{\prime}}\left(C_{12}+C_{21}-C_{11}-C_{22}\right)\right]$

The general solution to Equation (A22) is such that (Graziani and Ribacchi, 1993):

$$
\begin{gathered}
u=C_{1} \rho^{\alpha_{1}}+C_{2} \rho^{\alpha_{2}}-\frac{m\left(\sigma_{h}-\bar{\sigma}_{\rho \rho}\right) \rho}{E^{\prime} C_{11}\left(1-\alpha_{1}\right)\left(1-\alpha_{2}\right)} \\
\text { with } \alpha_{1,2}= \pm \frac{-\left(C_{12}-C_{21}\right) \pm \sqrt{\left(C_{12}-C_{21}\right)^{2}+4 C_{11} C_{22}}}{2 C_{11}}
\end{gathered}
$$

\footnotetext{
1 Equations (A14) and (A17) can be indifferently written using total stresses (for a purely elastic path, pore pressure is unaffected under undrained conditions).
} 
The two constants $C_{1}$ and $C_{2}$ are determined by expressing continuity of radial stress and radial displacement (they should be derivable) across the plastic boundary. Considering the linearity of the incremental law, these conditions are equivalent to:

$$
\rho=R_{p} \begin{cases}u=u^{e} & \bar{\varepsilon}_{\theta \theta}=\varepsilon_{\theta \theta} \\ \sigma_{\rho \rho}=\bar{\sigma}_{\rho \rho} & \mathrm{d} \sigma_{\rho \rho}=0 \quad \bar{\varepsilon}_{\rho \rho}=\varepsilon_{\rho \rho}\end{cases}
$$

Taking into account the compatibility equations (A21) one obtains:

$$
\begin{aligned}
& C_{1}=\frac{\bar{\varepsilon}_{\theta \theta}}{R_{p}^{\alpha_{1}-1}\left(\alpha_{2}-\alpha_{1}\right)}\left\lfloor\left(1+\alpha_{2}\right)-\frac{m}{C_{11}\left(1-\alpha_{1}\right)\left(1+v^{\prime}\right)}\right\rfloor \\
& C_{2}=\frac{-\bar{\varepsilon}_{\theta \theta}}{R_{p}^{\alpha_{2}-1}\left(\alpha_{2}-\alpha_{1}\right)}\left[\left(1+\alpha_{1}\right)-\frac{m}{C_{11}\left(1-\alpha_{2}\right)\left(1+v^{\prime}\right)}\right]
\end{aligned}
$$

By introducing the dimensionless variable $x=\rho / R_{p}$, the deformations take the form:

$$
\begin{aligned}
& \varepsilon_{\rho \rho}(x)=\bar{\varepsilon}_{\theta \theta}\left[\alpha_{1} F_{1}(x)-\alpha_{2} F_{2}(x)\right] \\
& \varepsilon_{\theta \theta}(x)=\bar{\varepsilon}_{\theta \theta}\left[F_{1}(x)-F_{2}(x)\right]
\end{aligned}
$$

with:

$$
\begin{aligned}
& F_{1}(x)=\frac{1}{\alpha_{2}-\alpha_{1}}\left[\left(1+\alpha_{2}\right) x^{\alpha_{1}-1}-\frac{m\left(x^{\alpha_{1}-1}-1\right)}{C_{11}\left(1+v^{\prime}\right)\left(1-\alpha_{1}\right)}\right] \\
& F_{2}(x)=\frac{1}{\alpha_{2}-\alpha_{1}}\left[\left(1+\alpha_{1}\right) x^{\alpha_{2}-1}-\frac{m\left(x^{\alpha_{2}-1}-1\right)}{C_{11}\left(1+v^{\prime}\right)\left(1-\alpha_{2}\right)}\right]
\end{aligned}
$$

Stresses in the plastic ring can now be easily calculated by replacing (A26) in (A10), i.e.:

$$
\begin{aligned}
& \sigma_{\rho \rho}=\bar{\sigma}_{\rho \rho}+\frac{1}{\operatorname{det}|\mathbf{A}|}\left[C_{11} \mathrm{~d} \varepsilon_{\rho \rho}+C_{12} \mathrm{~d} \varepsilon_{\theta \theta}\right] \\
& \sigma_{\theta \theta}=\bar{\sigma}_{\theta \theta}+\frac{1}{\operatorname{det}|\mathbf{A}|}\left[C_{21} \mathrm{~d} \varepsilon_{\rho \rho}+C_{22} \mathrm{~d} \varepsilon_{\theta \theta}\right]
\end{aligned}
$$

with:

$$
\begin{aligned}
& \mathrm{d} \varepsilon_{\rho \rho}=\varepsilon_{\rho \rho}-\bar{\varepsilon}_{\rho \rho}=\bar{\varepsilon}_{\theta \theta}\left[\alpha_{1} F_{1}(x)-\alpha_{2} F_{2}(x)+1\right] \\
& \mathrm{d} \varepsilon_{\theta \theta}=\varepsilon_{\theta \theta}-\bar{\varepsilon}_{\theta \theta}=\bar{\varepsilon}_{\theta \theta}\left[F_{1}(x)-F_{2}(x)-1\right]
\end{aligned}
$$

Finally, the plastic radius is calculated by expressing the well boundary condition, i.e.:

$$
\sigma_{\rho \rho}\left(x_{w}\right)=p_{w} \quad \text { with } x_{w}=\frac{R}{R_{p}}
$$

In the elastic zone (which extends from the plastic radius to infinity), constant $K_{2}$ (Eq. (A14)) is determined by expressing the continuity of the radial stress through the plastic boundary, i.e.:

$$
\rho=R_{p} \Rightarrow \sigma_{\rho \rho}=\bar{\sigma}_{\rho \rho}\left\{\begin{array}{l}
\sigma_{\rho \rho}=\left(1-\frac{R_{p}^{2}}{\rho^{2}}\right) \sigma_{h}+\bar{\sigma}_{\rho \rho} \frac{R_{p}^{2}}{\rho^{2}} \\
\sigma_{\theta \theta}=\left(1+\frac{R_{p}^{2}}{\rho^{2}}\right) \sigma_{h}-\bar{\sigma}_{\rho \rho} \frac{R_{p}^{2}}{\rho^{2}}
\end{array}\right.
$$

